\title{
A case of primary serous papillary carcinoma with unusual clinical presentation: distant lympho nodes metastasis without peritoneal dissemination
}

\author{
Toshie Yonehara • Tatsumi Yamaguchi • \\ Masaki Azuma $\cdot$ Shinichiro Minobe \\ Noriaki Sakuragi
}

Received: 12 December 2007 / Accepted: 26 February 2008 / Published online: 13 March 2008

(C) The Author(s) 2008

\begin{abstract}
Case report A 50-year-old woman with primary serous papillary carcinoma (PSPC) of peritoneal origin located in the posterior uterine serosa and cul-de-sac without peritoneal dissemination. No peritoneal dissemination was detected but the tumor metastasized to para-aortic and supraclavicular lymph nodes. After first chemotherapy course, pericardial effusion occurred. A pericardiectomy was performed to prevent cardiac failure. Subsequent chemotherapy with paclitaxel and carboplatin was effective against this tumor

Conclusion In general, a typical type of PSPC usually develops distant metastasis with diffuse peritoneal dissemination; the present case shows unusual clinical behavior.
\end{abstract}

Keywords Peritoneal carcinoma · Pericardial effusion . Pericardiectomy $\cdot$ T-J therapy

\section{Introduction}

Primary serous papillary carcinoma (PSPC), first reported by Swerdlow [1], is a tumor with numerous morphologic and clinical similarities to serous epithelial ovarian

T. Yonehara $(\bowtie) \cdot$ T. Yamaguchi $\cdot$ M. Azuma $\cdot$ S. Minobe Department of Obstetrics and Gynecology,

Kushiro Red Cross Hospital, 21-14 Shinei-cho,

Kushiro 860-0032, Japan

e-mail: ytoshie@kushiro.jrc.or.jp

\section{N. Sakuragi}

Department of Obstetrics and Gynecology,

Hokkaido University School of Medicine,

North 15, West 7, Kitaku, Sapporo 060-8638, Japan carcinoma (EOC) but with poor prognosis [2]. A concise statement criteria for the diagnosis of PSPC was developed by the Gynecologic Oncology Group (GOG) [3] and retrospectively revised by Bloss et al. [3].

In most cases of PSPC, extensive intraperitoneal spread could be detected at the time of diagnosis, even in the absence of evidence of primary ovarian tumors.

In this article, we described a case of PSPC with unusual clinical behavior, in which the primary tumor was localized in the pelvic peritoneum and directly metastasized to distant lymph nodes without intraperitoneal spread. The patient developed pericardial effusion, which may be related to lymph node enlargement rather than malignant pericarditis. Awareness of this unusual presentation and clinical course of PSPC would make the diagnosis more straightforward leading to a precise management.

\section{Case report}

A 50-year-old postmenopausal woman first visited our Department in January 2005 with a 3-month history of abdominal distension and alternate stool abnormality. She was healthy earlier.

Clinical examinations revealed significant enlargement of left supraclavicular (Fig. 4a), para-aorta (Fig. 4b), right obturator and left common lymph nodes.

Gastrointestinal investigations, including gastrointestinal scopy and colonoscopy were normal. Abdominopelvic computed tomography (CT) scan showed massive ascites in the upper abdomen and pelvis (Fig. 1a) and a cystic tumor with partially solid region was found in cul-de-sac (Fig. 1a). Laboratory studies demonstrated elevated serum levels of CA-125, 1,600 U/ml (normal range $<35 \mathrm{U} / \mathrm{ml}$ ) and CA72-4, $27.8 \mathrm{U} / \mathrm{ml}$ (normal range $<10 \mathrm{U} / \mathrm{ml}$ ). 
Fig. 1 Enhanced CT scan of pelvis. CT scan shows massive ascites and tumor behind uterus (arrow, a). Histological appearance of the pelvic tumors which are similar to those of lymph ducts in the ovary (b; HE stain, $\times 100)$. Histological appearance of the ovary.

Papillary adenocarcinoma cells form clusters in lymph ducts in ovary $(\mathbf{c} ;$ HE stain, $\times 40)$

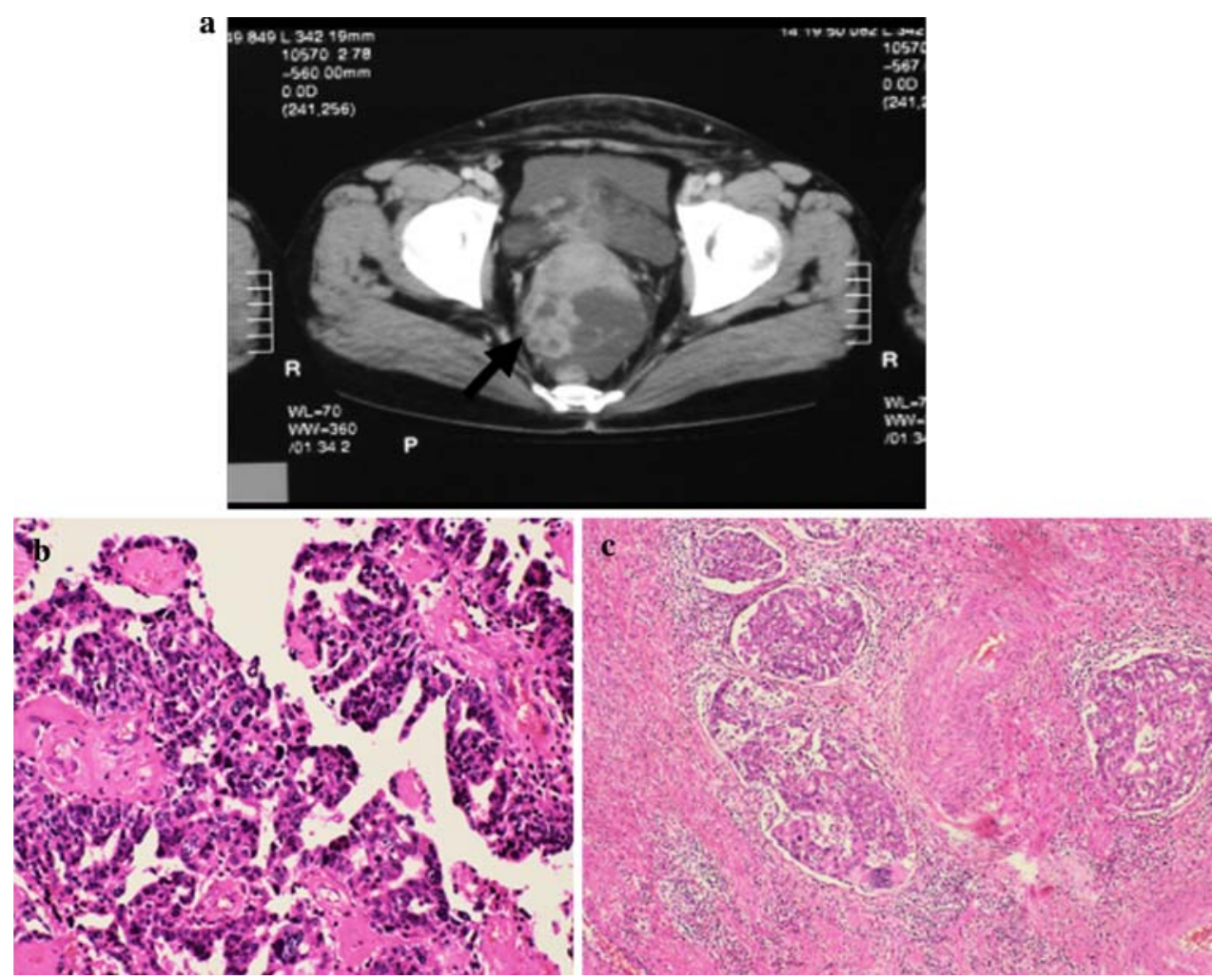

Abdominocentesis was performed in two occasions, on admission and after 3 days, with a removal of 21 of serous yellowish fluid each time. Cytological analysis of $20 \mathrm{ml}$ of ascitic fluid was performed in each occasion showing no abnormalities. The uterus cancer screening, both uterine body and cervix, was negative. The patient underwent exploratory laparotomy followed by total hysterectomy, bilateral salpingo-oophorectomy, appendectomy, partial peritoneal resection, and pelvic washings. A total of $1,500 \mathrm{ml}$ of ascitic fluid was removed at the entrance the peritoneal cavity, but there was no macroscopic tumoral dissemination. The cytology of the aspiratory biopsy was also negative.

Macroscopically, the uterus, bilateral tubes, and ovaries were normal in size and appearance. Only three completely excised masses were detected; one was located in the peritoneum of cul-de-sac $(1 \times 1 \mathrm{~cm})$, the second in the posterior wall of uterine corpus $(3 \times 2 \mathrm{~cm})$ and the third in the mesentery $(1 \times 1 \mathrm{~cm})$. These masses seemed to be derived from close peritoneal lesion. No macroscopic dissemination was observed in omentum.

Fast cytological analysis of ascites was negative; consequently, we did not perform omentectomy at that time. Subsequent histopathological examination revealed papillary serous carcinoma in the three masses (Fig. 1b). No psammonma bodies were found in these tumors. Similar histopathological findings were detected in lymph ducts of ovaries (Fig. 1c), not on the surface of ovaries indicating ovarian invasion of the papillary serous carcinoma via lymph ducts. Similar carci- noma cells were observed in the cardinal ligament but no in the uterine body. A histological diagnosis of papillary serous carcinoma of peritoneal origin was made, and defined at stage IV according to the 1987 FIGO classification.

As per patient's request, a further radical operation with pelvis and para-aorta lymphadenectomy, accompanied by biopsy of the distant lymph nodes, was not performed.

The first course of chemotherapy, with paclitaxel and carboplatin (TJ therapy), was started at the 20th postoperative day. Although there was a reduction on the levels of serum CA-125 to $43 \mathrm{IU} / \mathrm{ml}$, no effect was observed on the size of the lymph nodes. Four weeks after, a routine chest $\mathrm{X}$-ray showed cardiomegaly with cardiothoracic ratio $72 \%$ (Fig. 2a). Chest CT scan (Fig. 2b) and ultrasound cardiography (Fig. 2c) demonstrated severe pericardial effusion. She developed mild edema in her face and bilateral legs, but there was no dyspnea or palpitations. ECG was normal. With a presumptive diagnosis of cardiac tamponade due to continuous pericardial effusion, pericardiocentesis by ultrasound-guide was performed, removing $800 \mathrm{ml}$ of serous yellowish fluid. Laboratory analysis of the pericardial fluid showed $>100 /$ field white blood cells, mainly constituted of lymphocyte, and occult bacteria; however, no growth of bacterial or fungal was detected in cultured medium. No carcinoma cells were found in the pericardial fluid. The origin of the pericardial effusion could not be identified and we speculated that the enlarged lymph nodes disturbed lymphatic circulation leading to transudate effusion in pericardial cavity. 
Fig. 2 Chest X-ray shows cardiomegaly (a). Chest CT scan (b) and ultrasound cardiography (c) shows severe pericardial effusion

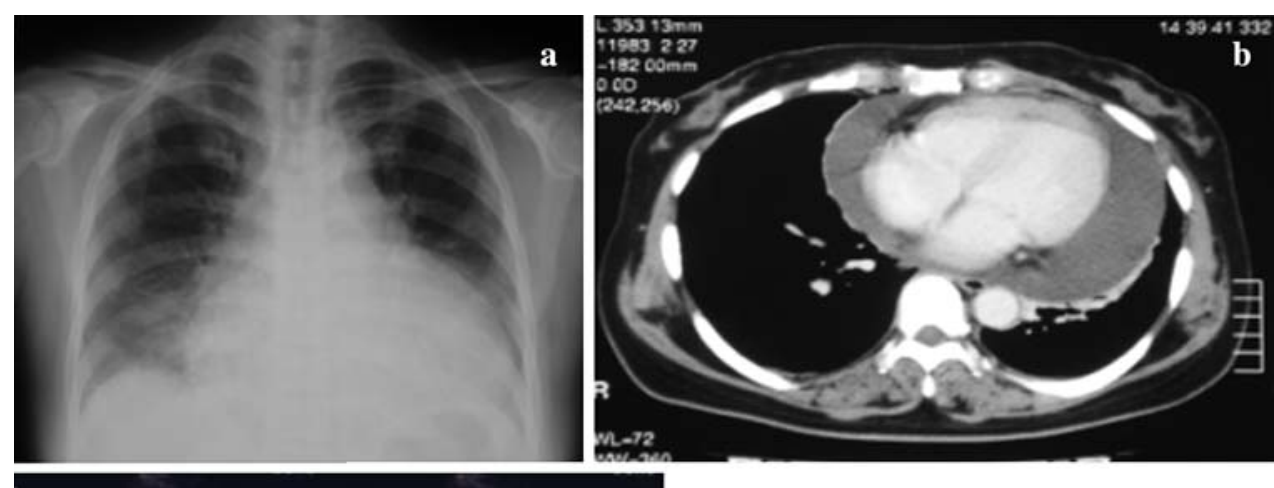

c

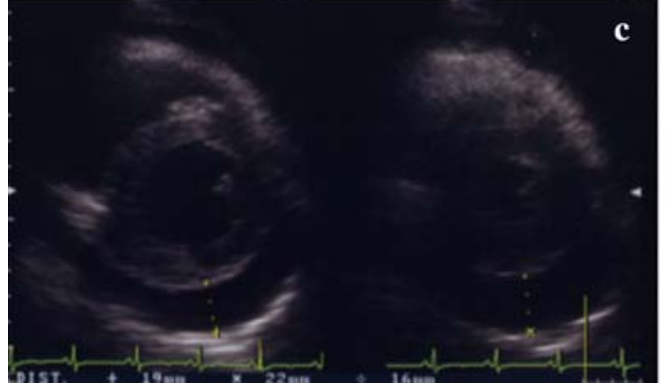

A second course of TJ therapy was initiated and was resumed before removing the pericardial fluid drainage because her condition was generally good. Three weeks after pericardiocentesis, the drainage tube was removed, but pericardial effusion recurred within 2 weeks. To prevent cardiac tamponade and pericardial sclerosis, the patient underwent pericardiectomy. No carcinoma cells were found in pericardial fluid at the time of pericardiectomy and pericardial effusion did not recur after pericardiectomy.

Subsequent four courses TJ therapy completed without any troubles leading to significant reduction of CA-125 serum levels (Fig. 3) and to the reduction of the enlarged supraclavicular and para-aorta lymph nodes to normal size (Fig. 4c, d). These findings suggested that the enlargement of the distant lymph nodes might be due to carcinoma metastasis. After a total of six courses of TJ therapy, performed every 3 weeks, monthly course of chemotherapy only with paclitaxel was continued for 12 months. With ongoing chemotherapy there was no clinical evidence of disease. When last seen on July 2007, she had no clinical finding of recurrence.

\section{Discussion}

Diffuse involvement of the peritoneum with massive ascites is one of the characteristics of the primary peritoneal malignant neoplasm, including PSPC [4].

We present a case of PSPC with a rare complication derived from lymph node metastasis. The carcinoma was only localized on cul-de-sac without spreading in the peritoneal cavity, and although there were massive ascites, no

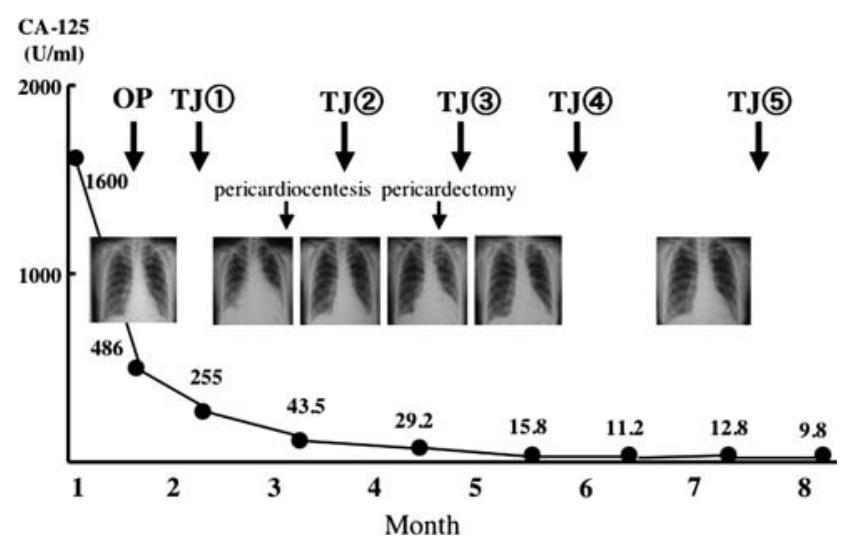

Fig. 3 Change of serum CA-125 levels and pericardial effusion along the course of treatment ( $O P$ operation, $T J \mathrm{TJ}$ therapy)

carcinoma cell was detected by repeated aspiratory cytology of the fluid.

The most remarkable aspect of our case is its spread manner. There was a significantly enlargement of pelvis and para-aorta lymph nodes, as well as supraclavicular distant nodes in the absence of peritoneal dissemination, a characteristic that is rarely found in PSPC [4]. Only a few cases of PSPC with distant metastasis without peritoneal dissemination have been published. Miyaishi et al. [5] reported one case of metastasis to pleura and hilar lymph nodes without peritoneal dissemination in PSPC.

The development of pericardial effusion in PSPC is also extremely rare. We found no reports on PSPC with pericardial effusion without peritoneal dissemination by a Medline search from 1966 to 2006. Even in ovarian cancer, pericarditis is a very rare complication and little 
Fig. 4 Change of lymph nodes enlargement with treatment (arrow). Before treatment CT scan of neck and upper abdomen shows enlarged subclavicular lymph node (a) and para-aortic lymph node (b), respectively. After treatment subclavicular lymph node (c) and para-ovarian lymph node (d) are reduced

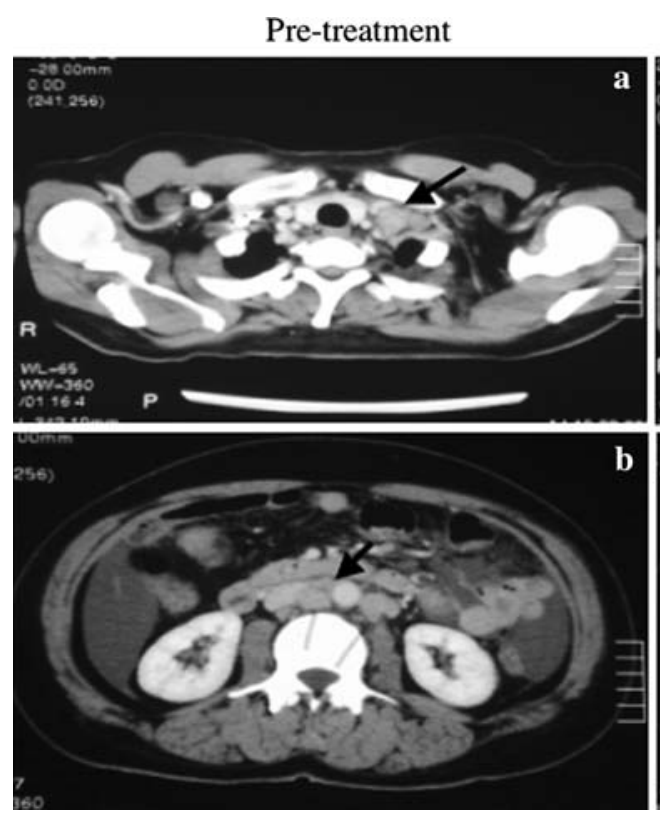

Post-treatment

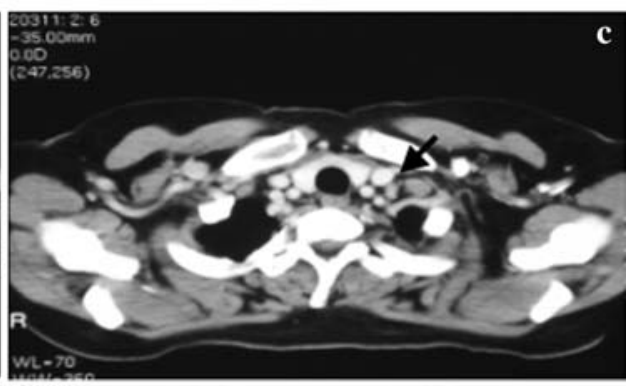

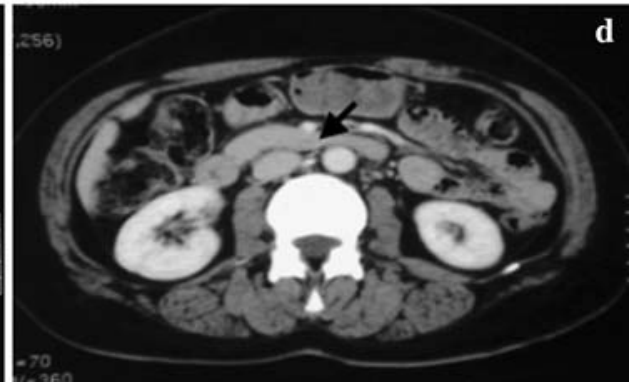

information is published [6]. Donato et al. [7] reported a case of EOC metastasizing to the heart and/or pericardium associated with grossly advanced intraperitoneal cavity. Other cases of EOC that developed pericardial effusion were associated with pericardial metastasis or malignant pericarditis $[6,8]$, one of them caused pericardial tamponade [8].

In the present case, there was no macroscopic dissemination in both peritoneum and pericardium. The aspiratory cytology showed no carcinoma cells in any of the fluids. The aspirated pericardial fluid was mainly constituted of lymphocyte and no bacteria were detected. The pericardial fluid might be a transudate from congested lymphatic circulation rather than an exudate by pericarditis. It is possible that the enlargement of upper abdominal lymph nodes might disturb lymphatic circulation along lymph duct or venous flow.

Cardiac tamponade is a life-threatening condition usually induced by pericardial sclerosis associated with continuous pericardial effusion. Pericardiectomy is one of the possible treatments for this complication and it has been reported a 4-year survival after pericardiectomy in one case of recurrent malignant pericardial effusion associated with ovarian carcinoma [9]. In the present case, pericardiectomy was followed by chemotherapy due to the recurrence of pericardial effusion.

Treatment of PSPC is similar to that of EOC, which includes cytoreductive surgery and platinum-based combination chemotherapy. The serum levels of CA-125 may be useful for the evaluation of the effect of treatment. We treated our patient with six courses of paclitaxel and carboplatin (TJ therapy) after primary surgery with significantly reduction in the serum levels of CA-125 (Fig. 3). These findings suggest that chemotherapy was effective to reduce lymph nodes enlargement, improving the lymphatic circulation, and consequently leading to the resolution of the pericardial effusion.

Unfortunately, we could not perform adequate surgical staging on our patient. The lymph nodes were evaluated only by CT scan; therefore, we could not confirm the presence of metastasis at these locations, and other causes of lymph nodes enlargement could not be ruled out. However, the significant reduction of the lymph nodes size after TJ therapy with no recurrence, and the absence of inflammatory changes in the general laboratory examinations suggested that these enlargements of lymph nodes should be related to cancer metastasis, and lead to diagnose this patient as stage IV using the FIGO classification.

Although in EOC, a radical operation including lymphadenectomy and cytoreduction is effective even in advanced stage, it is still not clarified the therapeutic value of lymphadenectomy in PSPC. Dubernard et al. [10] showed that overall survival of patients with PSPC is similar to that of their EOC counterparts and that lymphadenectomy is not only an important diagnostic procedure but has also a predictive prognostic value PSPC [10].

In conclusion, our case shows the effectiveness of TJ therapy for PSPC without radical operation. Almost all the previous reports on PSPC with distant metastasis showed aggressive dissemination of peritoneum or pericardium with extremely poor prognosis. Additional case reports are required to decide the appropriate therapy, radical surgery, or systemic chemotherapy for distant metastasis without dissemination in PSPC. 
Open Access This article is distributed under the terms of the Creative Commons Attribution Noncommercial License which permits any noncommercial use, distribution, and reproduction in any medium, provided the original author(s) and source are credited.

\section{References}

1. Swerdlow M (1959) Mesothelioma of the pelvis peritoneum resembling papillary cystadenocarcinoma of the ovary. Am J Obstet Gynecol 77:197-200

2. Mills SE, Andersen WA, Fechner RE, Austin MB (1988) Serous surface papillary carcinoma: a clinicopatholigic study of 10 cases and comparison with stage III-IV ovarian serous carcinoma. Am J Surg Pathol 12:827-834

3. Bloss JD, Liao SY, Buller RE, Manetta A, Berman ML, McMeekin S, Bloss LP, DiSaia PJ (1993) Extraovarian peritoneal serous papillary carcinoma: a case-control retrospective comparison to papillary adenocarcinoma of the ovary. Gynecol Oncol 50:347-351

4. Truong LD, Maccato ML, Awalt H, Caqle PT, Schwarts MR, Kaplan AL (1990) Serous surface carcinoma of the peritoneum. Hum Pathol 21:99
5. Miyaishi O, Iida K, Saga S, Sato T (1994) An autopsy case of serous papirally carcinoma of peritoneum with distant metastases but no peritoneal dissemination. Gynecol Oncol 55:448-452

6. Bruzzese V, Biferali F, Paventi S (1994) Neoplastic pericarditis secondary to ovarian adenocarcinoma: report of clinical case. Minerva Med 85:607-10

7. Donato DM, Sevin BU, Averette HE (1986) Ovarian adenocarcinoma complicated by malignant pericarditis. Gynecol Oncol 24:171-176

8. Winter WE III, Seidman J, Krivak TC, Pujari SG, Boice CR, Carlson JW (2002) Papillary serous adenocarcinoma of the ovary diagnosed after malignant pericardial tamponade and embolic stroke. Gynecol Oncol 84:453-455

9. Duflo B, Durigon M, Guerre J, Hazan E, Lemant P, Pequignot H (1973) Reccurent hemorrhage pericarditis revealing adenocarcinoma of probable ovarian origin. 4-year survival following pericardectomy. Semin Hosp Paris 49:3459-3462

10. Dubernard G, Morice P, Rey A, Camatte S, Fourchotte V, Thoury A, Pomel C, Pautier P, Lhomme C, Duvillard P, Castaigne D (2004) Prognosis of stage III or IV primary peritoneal serous papillary carcinoma. Eur J Surg Oncol 30:976-981 